\title{
COOPERATIVE MIMO TRANSMISSIONS IN WSN USING THRESHOLD BASED MAC PROTOCOL
}

\author{
J. Vidhya ${ }^{1}$ and P. Dananjayan ${ }^{2}$ \\ Department of Electronics and Communication Engineering \\ ${ }^{1}$ Rajiv Gandhi College of Engineering and Technology, Puducherry, INDIA \\ ${ }^{2}$ Pondicherry Engineering College, Puducherry, INDIA \\ pdananjayan@rediffmail.com
}

\begin{abstract}
Sensor networks require robust and efficient communication protocols to maximise the network lifetime. Radio irregularity, channel fading and interference results in larger energy consumption and latency for packet transmission over wireless channel. Cooperative multi-input multi-output (MIMO) schemes when incorporated in wireless senor network (WSN) can significantly improve the communication performance. An inefficiently designed medium access control (MAC) protocol however, may diminish the performance gains of MIMO operation. Hence, this paper proposes a distributed threshold based MAC protocol for cooperative MIMO transmissions using space time block codes (STBC). The protocol uses a thresholding scheme that is updated dynamically based on the queue length at the sending node to achieve lesser energy consumption and minimise latency ensuring the stability of transmission queues at the nodes. STBC and code combining techniques are applied to utilise the inherent spatial diversity in wireless cooperative MIMO systems. Simulation results are provided to evaluate the performance of the proposed protocol and are compared with fixed group size cooperative MIMO MAC protocols with and without STBC coding. Results show that the proposed protocol outperforms point to point communication as well as cooperative MIMO MAC protocols that use fixed group sizes. STBC technique for the proposed $M A C$ protocol provides significant energy savings and minimises the packet delay by leveraging MIMO diversity gains.
\end{abstract}

\section{KEYWORDS}

Cooperative MIMO, energy efficiency, MAC protocol, STBC, thresholding scheme, wireless sensor network

\section{INTRODUCTION}

Wireless sensor network (WSN) comprises of hundreds to thousands of small nodes employed in a wide range of data gathering applications such as military, environmental monitoring and other fields [1]. Due to limited energy and difficulty in recharging a large number of sensor nodes, energy efficiency and maximising network lifetime have been the most important design goals for the network. However, channel fading and radio interference pose a big challenge in design of energy efficient communication protocols for WSN.

To reduce the fading effects in wireless channel, multi-input multi-output (MIMO) scheme is utilised for sensor network [2,3]. MIMO systems can dramatically increase the channel capacity and reduce the transmission energy in wireless fading channels. Sensor nodes using MIMO techniques would require lower transmission power to achieve the same bit error rate (BER) as point to point communications [4]. Applying multiple antenna techniques directly to sensor network is impractical because of the limited size of a sensor node usually supports a single antenna.

Cooperative transmission and reception from antennas in a group of sensor nodes can be used to construct a system fundamentally equivalent to a MIMO system for WSN [5]. Normally, MIMO 
system needs to estimate all channels between source and destination. If cooperative transmissions from multiple sensor nodes are allowed, the amount of channel estimation at the receiver can be reduced and hence can save the energy of sensor nodes [4,5]. The complexity in coordinating the actions of sensor nodes limits the practical use of MIMO in WSN. Also, an inefficiently designed medium access control (MAC) protocol will increase the energy spent in exchanging the cooperative control messages, and diminish the performance of MIMO system.

The fundamental task of a MAC protocol [6] is to schedule the transmissions from nodes sharing the same channel and prevents collisions. Due to energy constraints in WSNs, protocols for WSNs have the additional requirement to be energy efficient. Most current MAC protocols for WSNs use sleep-wake cycles to reduce the energy wastage during idle listening. However, sleep-wake cycles may be inappropriate for time critical applications because of long packet delays.

MAC protocols can be classified as either contention based or collision free. The most popular contention based MAC protocol is SMAC [6]. In SMAC each node follows a sleep-wake cycle to reduce the energy consumption. For collision free MACs, centralised architecture is widely used [7]. However, the use of centralised architecture [7-11] for cooperative MIMO MAC transmissions leads to energy wastage on cluster maintenance and introduces additional coordination delays.

To minimise the energy wastage, distributed system architecture [12-13] for cooperative MIMO MAC transmission [14] is utilised. In this protocol, the source and destination nodes cooperate with their neighbouring nodes while transmitting and receiving data. The MIMO transmission system achieves lower overall energy consumption than point to point communications [2]. However, the number of nodes in the sending and receiving groups is fixed and is difficult to set the right numbers for the groups to achieve the minimum energy consumption and delay and increases the likelihood that the queue length at the sender becomes unstable [12-13].

To address these issues and facilitate cooperative MIMO transmissions with a high degree of performance improvement, a new MAC protocol is suggested for scheduling cooperative MIMO transmissions in distributed WSNs. The proposed MAC protocol dynamically selects the cooperative group size based on the thresholding scheme. The cooperative threshold is updated by the receiver based on the queue length at the source and the number of neighbours recruited at the sending node. This threshold is essential to maintain maximum throughput and increase the network lifetime. If the desired threshold is achieved, the destination node calculates the size of sending and receiving groups that has minimum energy consumption to proceed with MIMO data transmission. The proposed MIMO MAC protocol utilises space time block code (STBC) scheme [15-17] and provides significant diversity gain to enhance the system performance. This protocol outperforms fixed group size cooperative MIMO MAC and point to point communication schemes in terms of energy and delay.

The remainder of the paper is organised as follows. Section 2 presents the proposed cooperative MIMO MAC model. In section 3 the mathematical model to analyse the performance of the proposed MAC protocol is presented. Simulation results are discussed in section 4 to evaluate energy consumption and delay of the proposed MAC scheme utilising STBC coding and conclusions are drawn in section 5 .

\section{SYSTEM MODEL}

In cooperative MIMO systems, transmit and receive diversity are achieved in a distributed manner by the sending and receiving group/cluster [12-13]. In the sending group, transmitted signals from multiple sending nodes are combined before arriving at the receiver. The proposed cooperative MIMO communication strategy consists of three steps and is shown in Fig.1.

\section{i) Broadcasting}


The source node broadcasts its data using low transmission power to the selected source cluster members and destination as shown in Fig.1a. The selection of cluster members is based on the space time block coding requirement. The source node specifies the order for selected cooperative nodes so that each cooperative node will choose one of the rows of STBC [15-17] code for cooperative MIMO data transmission.

\section{ii) STBC MIMO transmission}

As shown in Fig.1b, the cooperative nodes in sending group will use the corresponding row of STBC code, assigned in step 1, to change the permutation of data bits. Then, all nodes in the sending group, including the source node, will transmit space-time block coded data to the receiving group. Multiple nodes in the sending and receiving group form cooperative system to achieve MIMO diversity.

\section{iii) Data collection and combining}

After receiving data from the sending group as shown in Fig.1c, each node in the receiving group uses the channel state information to decode the space-time block coded data. After decoding the STBC, cooperative nodes in receiving group relay their copies to the destination node. The destination receives signal copies from the cooperative nodes and detects them as soft symbols. It then uses code combining and chooses the most possible codeword based on the received soft symbols.

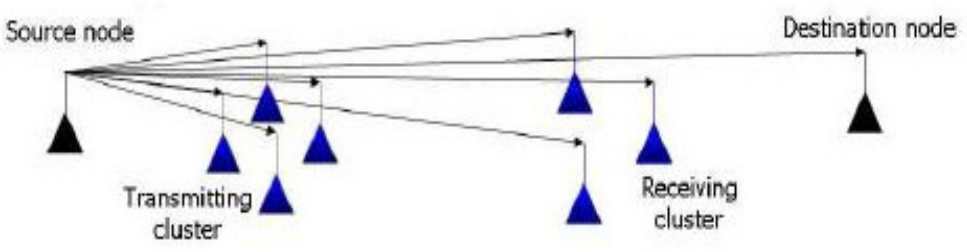

(a). Broadcasting

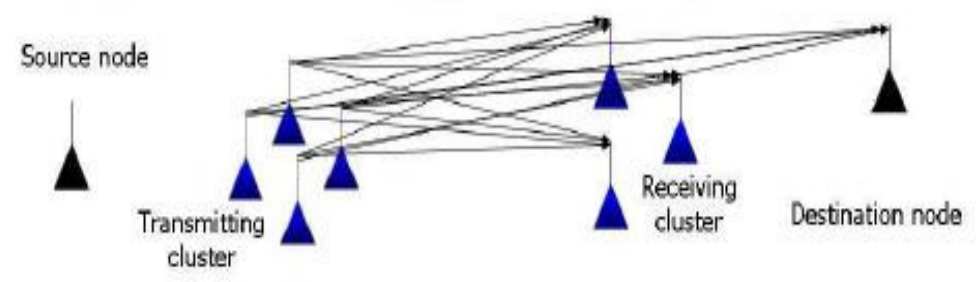

(b). MIMO transmission
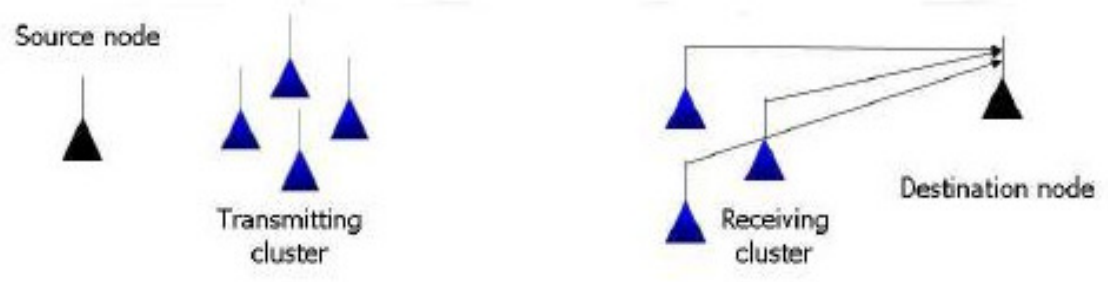

(c). Data collection and combining

Fig.1. Proposed cooperative scheme 


\subsection{Proposed Cooperative MIMO MAC Protocol}

The proposed cooperative MIMO MAC protocol for coordinating transmissions from multiple nodes is discussed below. Consider the operation of source node that forwards a packet to destination as shown in Fig.2.

When a node has data to send, it first senses the channel to ensure that it is idle. If the channel is sensed to be busy, the node initialises a backoff timer and waits for the idle channel. If the backoff timer has decremented to zero, the source node first broadcasts recruiting message at low transmission power to its local neighbours for cooperative transmission $[6,14]$.

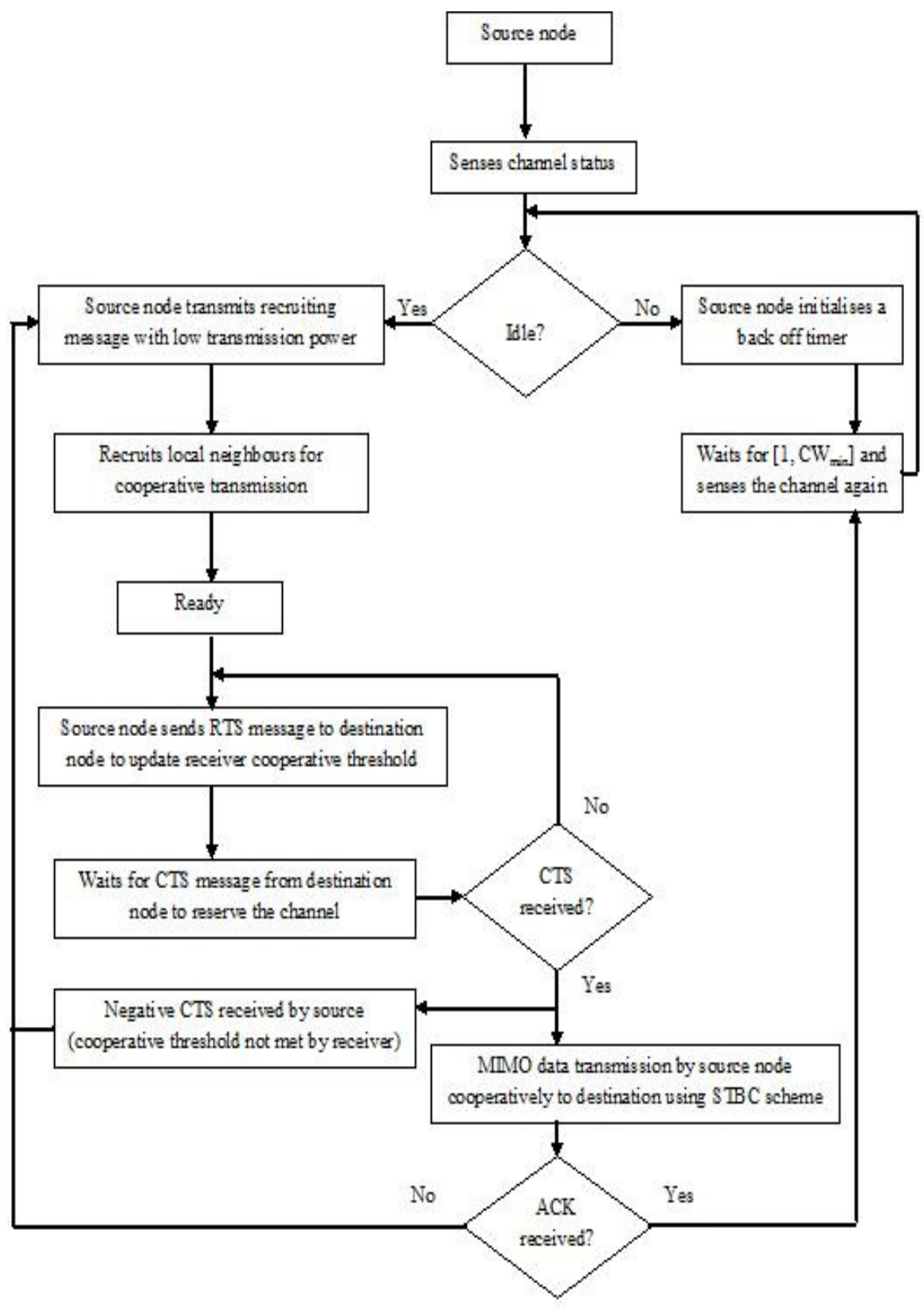

Fig.2. Flow chart of cooperative threshold based MAC protocol for source node 
When the replies are received from the neighbours, the source node transmits a request-to-send (RTS) message to destination at normal power. It then waits for the clear-to-send (CTS) reply from destination node to reserve the channel for data transmission. The RTS message contains information on the current queue length at the sender and the number of neighbours it has recruited. This information is used by the receiver to update the cooperative threshold.

The source node receives a negative CTS (NCTS) packet from the destination node as shown in Fig. 3 if the receiver is unable to update the cooperative threshold. During this process, the source node will backoff, recruit the cooperative nodes and attempt for retransmission. When the source does not receive the CTS packet within the specified time interval, the node automatically attempts for retransmission.

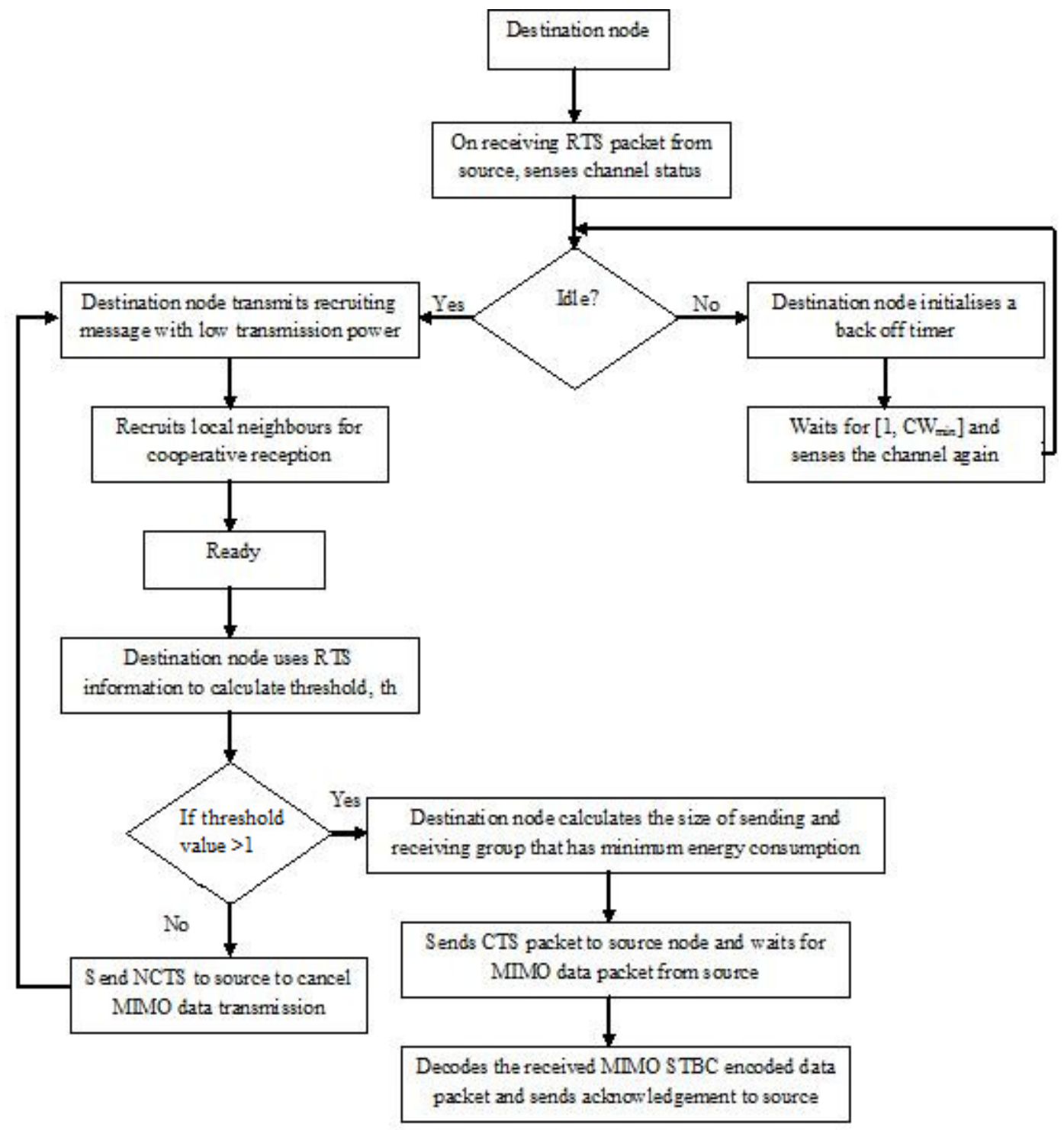

Fig.3. Flow chart of cooperative threshold based MAC protocol for destination node 
Once the CTS packet is received, the source node proceeds with the data transmission. Each CTS packet contains the optimum size of the cooperative group at the sending end. The source node broadcasts the data packet at low power to the nodes in its group and synchronises them.

Each node in the source-cluster transmits the data cooperatively using STBC coding $[4,5,7,8]$ and waits for an acknowledgement (ACK) from the destination node. If no ACK is received the retransmission process begins starting from neighbour recruitment.The operation of the destination node is as shown in Fig.3.

The destination node on receiving the RTS packet, seeks for an idle channel. If the channel is idle, the destination node sends a recruiting packet at low power to recruit its neighbours. On receiving replies from nodes willing to cooperate for reception, the destination node uses the RTS packet to calculate the threshold. The determination of cooperative threshold is described in section 2.2.

If the cooperative threshold as required is not met, a NCTS packet is sent to source node to cancel the transmission. On the other hand, if the threshold is met, the destination node broadcasts a low power message to the cooperative receiving group to help in the reception. It then sends a CTS packet with the required cooperative group size to source node.

The destination node waits for data transmission from the source cluster. Next, it waits for each node in the destination cluster to sequentially forward its copy of the received data packet. Finally it decodes the packet by combining all copies of the received packet and replies with an ACK packet to the source if the packet is decoded correctly. Otherwise, the destination node does nothing and the source node will eventually timeout.

\subsection{Proposed Thresholding Scheme}

The destination node uses the RTS information i.e., queue length and available cooperative node at the sender to calculate the threshold. The methodology to determine the threshold for the proposed MAC protocol is shown in Fig.4. Consider the source and destination cluster sizes available for cooperation to be $\mathrm{M}$ and $\mathrm{N}$ respectively. For each possible choice of $\mathrm{M}, \mathrm{N}$, the expected packet error rate (PER), $\mathrm{P}_{\mathrm{e}}(\mathrm{M}, \mathrm{N})$ is first evaluated using STBC coding [16].

Let the number of unique PER values obtained for the possible choices of cluster sizes are denoted by $\mathrm{K}$. The $\mathrm{K}$ successful packet transmission probabilities i.e., $\varphi(\mathrm{i})=\varphi(1), \varphi(2), \ldots$, $\varphi(K)$ are listed and their corresponding cluster sizes are derived. When the current queue length at the sender is $\mathrm{Q}$, threshold i, i.e., $\varphi(\mathrm{i})$ in terms of the desired successful packet transmission, is chosen if $(\mathrm{K}-\mathrm{i}) \xi<\mathrm{Q} \leq(\mathrm{K}-\mathrm{i}+1) \xi$, where $\xi$ is a positive integer. The threshold is set at 1 for $\mathrm{Q}>$ $\mathrm{K} \xi$. For threshold i choosen, the possible set of $\mathrm{S}=(\mathrm{M}, \mathrm{N})$ cluster sizes is obtained for which the packet delivery rate is greater than $\varphi(i)$.

The destination node does an exhaustive search of the possible group sizes of $\mathrm{M}, \mathrm{N}$ of $\varphi(\mathrm{i})$ and selects the combination that has the lowest energy consumption subject to the threshold. The cooperative group size $\mathrm{M}, \mathrm{N}$ corresponding to this energy consumption is dynamically selected as sending and receiving group for data transmission.

\section{Performance Analysis of Proposed Cooperative mimo maC MODEL}

A mathematical model to evaluate error probability, packet delay and energy consumption for the proposed cooperative MIMO MAC transmission scheme is described below. The bit error rate is assumed to be 0 in the first step (broadcasting) since a node can be in the sending group only if it receives the data packet correctly. Thus the bit error rate performance for transmission of data from transmit cooperative nodes to receive cooperative nodes and the bit error rate performance after code combining in the destination node have to be considered. The bit error 
probability is used to analyse the system energy consumption and the delay incurred in the transmission of data from the source to the destination.

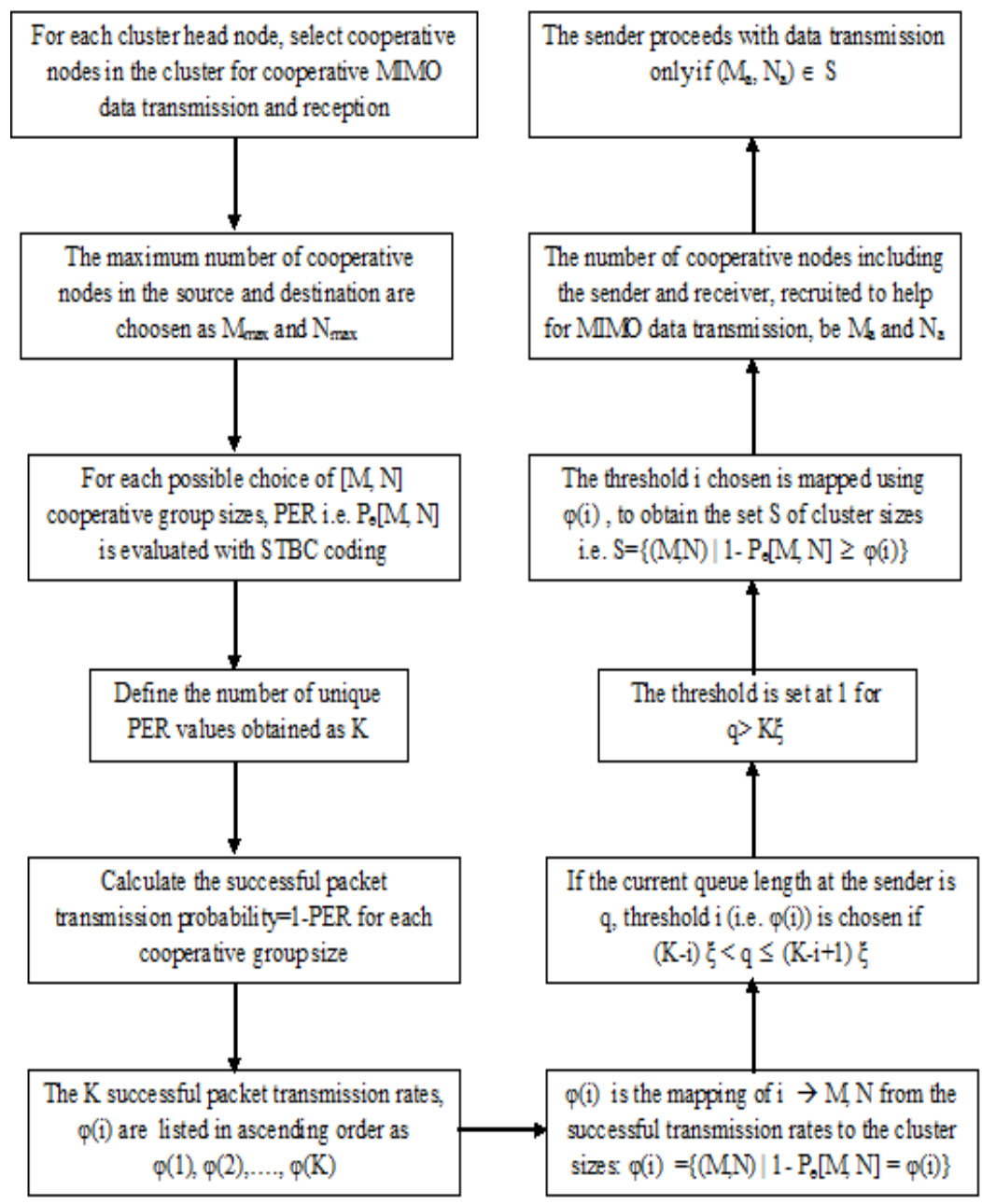

Fig.4. Flowchart of thresholding scheme for the proposed MAC protocol

\subsection{Bit Error Probability}

The system is assumed to transmit quadrature phase shift keying (QPSK) signals [2] through Rayleigh fading channel with additive white Gaussian noise (AWGN) noise. The relationship between the packet error probability $\mathrm{p}_{\mathrm{p}}$ and bit error probability $\mathrm{p}_{\mathrm{b}}[3,12]$ is given by

$\mathrm{p}_{\mathrm{p}}=1-\left(1-\mathrm{p}_{\mathrm{b}}\right)^{\mathrm{L}}$

where

$\mathrm{L}$ is the frame length in bits

Data transmission errors are generated from two factors in cooperative MIMO i.e. from the sending group to the receiving group and from cooperative receiving nodes to the destination. Since the cooperative sending nodes will not forward the data packet when it is corrupted, the error from the source to its neighbours will not be considered. 


\subsection{Energy Consumption Analysis}

Consider a scenario with $\mathrm{M}$ senders and $\mathrm{N}$ receivers involved in cooperative MIMO transmission. The energy consumed for an unsuccessful transmission attempt and for a successful transmission from sending to the receiving group using STBC MIMO MAC are calculated to analyse the overall energy consumption in a hop $[2,13]$.

The energy consumption for an unsuccessful transmission attempt is

$$
\mathrm{Eu}_{\text {coop }}=\mathrm{E}_{\text {mrts }}+\mathrm{E}_{\text {mcts }}+2 \mathrm{E}_{\text {rrts }}+(\mathrm{M}-1) \mathrm{E}_{\text {scts }}+(\mathrm{N}-1) \mathrm{E}_{\text {scts }}+\mathrm{E}_{\mathrm{br}}+\mathrm{E}_{\text {data }}+(\mathrm{N}-1) \mathrm{E}_{\text {col }}
$$

and the energy consumption for a successful attempt is

$$
\begin{aligned}
\mathrm{Es}_{\text {coop }} & =\mathrm{E}_{\text {mrts }}+\mathrm{E}_{\text {mcts }}+2 \mathrm{E}_{\text {rrts }}+(\mathrm{M}-1) \mathrm{E}_{\text {scts }}+(\mathrm{N}-1) \mathrm{E}_{\text {scts }} \\
& +\mathrm{E}_{\mathrm{br}}+\mathrm{E}_{\text {data }}+(\mathrm{N}-1) \mathrm{E}_{\text {col }}+\mathrm{E}_{\text {ack }}
\end{aligned}
$$

where

$\mathrm{E}_{\mathrm{mrts}}$ is the energy consumed in sending MIMO RTS (MRTS)

$\mathrm{E}_{\text {mcts }}$ is the energy consumed in sending MIMO CTS (MCTS)

$\mathrm{E}_{\mathrm{ack}}$ is the energy consumed in sending ACK

$\mathrm{E}_{\text {rrts }}$ is the energy consumed in sending recruiting RTS (RRTS)

$\mathrm{E}_{\text {scts }}$ is the energy consumed in sending sequential CTS (SCTS)

$\mathrm{E}_{\mathrm{col}}$ is the energy consumed by data collection in the third phase

$E_{b r}$ is the energy consumption of broadcasting data to cooperative nodes in sending group

$E_{\text {data }}$ is the energy consumption for data transmission between sending and receiving group

The MRTS and MCTS messages are control messages between source and destination and they require higher transmission power for long distance transmission. The RRTS and SCTS are control messages between source/destination and their neighbours. Compared to the MIMO RTS and CTS, RRTS and SCTS can be transmitted with less power due to short-distance transmission. In the receiving group, each node will transmit its signal back to the destination with energy $\mathrm{E}_{\mathrm{col}}$ and there are $\mathrm{N}-1$ cooperative nodes in the receiving group, excluding the destination node.

The total energy consumption for every one-hop transmission in cooperative MIMO system [18] is given by

$$
\mathrm{E}_{\mathrm{M}}=\frac{\mathrm{p}_{\mathrm{M}}}{\left(1-\mathrm{p}_{\mathrm{M}}\right)} \mathrm{Eu}_{\mathrm{coop}}+\mathrm{Es}_{\mathrm{coop}}
$$

where

$\mathrm{p}_{\mathrm{M}}$ is the packet error probability obtained considering uncoded or STBC scheme

Based on the above analysis, the destination node in the proposed protocol determines the sending and receiving group sizes. With the given number of nodes available at the sending and receiving groups, the destination node determines an exhaustive search of the possible sizes of $\mathrm{M}, \mathrm{N}$ and selects the combination that has lower energy consumption, subject to the threshold for MIMO data transmission.

\subsection{Packet Transmission Delay}

Each packet transmission in cooperative MIMO requires more steps as shown in Figs.2 and 3, which may increase the packet delays. However, the reduction in the packet error probability with cooperative MIMO MAC reduces the occurrence of retransmissions which in turn reduces the packet delays in comparison to point to point MAC protocols $[6,11,18]$. 
The duration of transmission attempt that is successful using cooperative MIMO transmission is given by

$\mathrm{Ts}_{\text {coop }}=\mathrm{T}_{\text {rts }}+\mathrm{T}_{\mathrm{Br}}+\mathrm{T}_{\text {cts }}+\mathrm{T}_{\mathrm{Bs}}+\mathrm{T}_{\text {data }}+\mathrm{T}_{\text {col }}+\mathrm{T}_{\text {ack }}$

and the duration for an unsuccessful attempt is

$\mathrm{Tu}_{\text {coop }}=\mathrm{T}_{\mathrm{rts}}+\mathrm{T}_{\mathrm{Br}}+\mathrm{T}_{\text {cts }}+\mathrm{T}_{\mathrm{Bs}}+\mathrm{T}_{\text {data }}+\mathrm{T}_{\text {col }}+\mathrm{T}_{\text {wait }}$

where

$\mathrm{T}_{\mathrm{rts}}$ is the transmission time for the RTS

$\mathrm{T}_{\mathrm{cts}}$ is the transmission time for the CTS

$\mathrm{T}_{\text {ack }}$ is the transmission time for the ACK

$\mathrm{T}_{\mathrm{data}}$ is the transmission time for the data

$\mathrm{T}_{\text {wait }}$ is the duration for which sender waits for an ACK

$\mathrm{T}_{\mathrm{Br}}$ is the transmission time of a recruitment message sent by the destination node

$\mathrm{T}_{\mathrm{Bs}}$ is the transmission time required for the source node to send the data packet to its cooperating nodes

$\mathrm{T}_{\mathrm{col}}$ is the time required by the cooperating receiving nodes to send the data to the destination

The total expected packet delay for cooperative MIMO MAC is given by

$$
\mathrm{T}_{\mathrm{dM}}=\frac{\mathrm{p}_{\mathrm{M}}}{\left(1-\mathrm{p}_{\mathrm{M}}\right)} \mathrm{Tu}_{\mathrm{coop}}+\mathrm{Ts}_{\mathrm{coop}}
$$

For the dynamically selected group size with thresholding scheme, the delays are evaluated taking into account STBC error probability.

\section{Simulation RESUlts}

The analysis of cooperative MIMO MAC protocol is carried out using MATLAB. The parameters considered for simulation is summarised in Table 1. The performance of proposed threshold based cooperative MIMO MAC protocol with STBC and uncoded schemes are evaluated in terms of energy consumption and delay incurred in the transmission of data packets from source to the destination node.

Table 1. Simulation parameters

\begin{tabular}{|l|l|}
\hline Parameter & Value \\
\hline Total frames per packet & 10 frames \\
\hline Total bytes per packet & 410 bytes \\
\hline Time for transmitting RTS & $35.3 \mathrm{~ms}$ \\
\hline Time for transmitting CTS & $30.5 \mathrm{~ms}$ \\
\hline Time for transmitting ACK & $32 \mathrm{~ms}$ \\
\hline Time for transmitting data & $0.006 \mathrm{~s}$ \\
\hline $\begin{array}{l}\text { Energy consumed for transmission } \\
\text { of RTS, CTS and ACK }\end{array}$ & $0.027 \mathrm{~J}$ \\
\hline $\begin{array}{l}\text { Energy consumed for transmission } \\
\text { of data }\end{array}$ & $0.2 \mathrm{~J}$ \\
\hline Modulation type & QPSK \\
\hline Channel & $\begin{array}{l}\text { Rayleigh fading } \\
\text { channel }\end{array}$ \\
\hline
\end{tabular}




\subsection{Performance Analysis of Uncoded MIMO Scheme}

The energy consumption for various diversity orders for the uncoded system for the proposed MAC protocol is shown in Fig.5. For lesser cooperative sending and receiving group sizes, symbol error rate (SER) increases at low signal-to-noise ratio (SNR), which results in multiple retransmissions, thereby resulting in higher power consumption of sensor node. When the SNR is increased, SER is reduced and hence the energy consumption can be decreased. The energy consumption is lesser when 4 cooperative nodes are used at transmit and receive clusters. It consumes $10 \%$ less energy than point to point scheme. This reduction in energy consumption is due to diversity gain of cooperative MIMO systems.

It is also observed from the graph that the proposed scheme outperforms fixed group size MIMO scheme by changing the cooperative threshold according to the queue length at the sender. The dynamic group size selected using cooperative threshold scheme is $4 \mathrm{x} 4$ MIMO configuration as it minimises the energy expended on recruiting and time spent on waiting for the required number of nodes in retransmission. The delay incurred for various transmit and receive group sizes are plotted in Fig.6. The delay keeps reducing with the increase in diversity gain due to increase in the number of receiving cooperative nodes. The decrease in delay is due to less SER and fewer retransmissions in the system. It is clear that the proposed scheme chooses the dynamic group size $4 \times 4$ based on cooperative threshold as it has fewer data retransmissions and results in $4 \%$ lesser packet latency than without MIMO scheme.

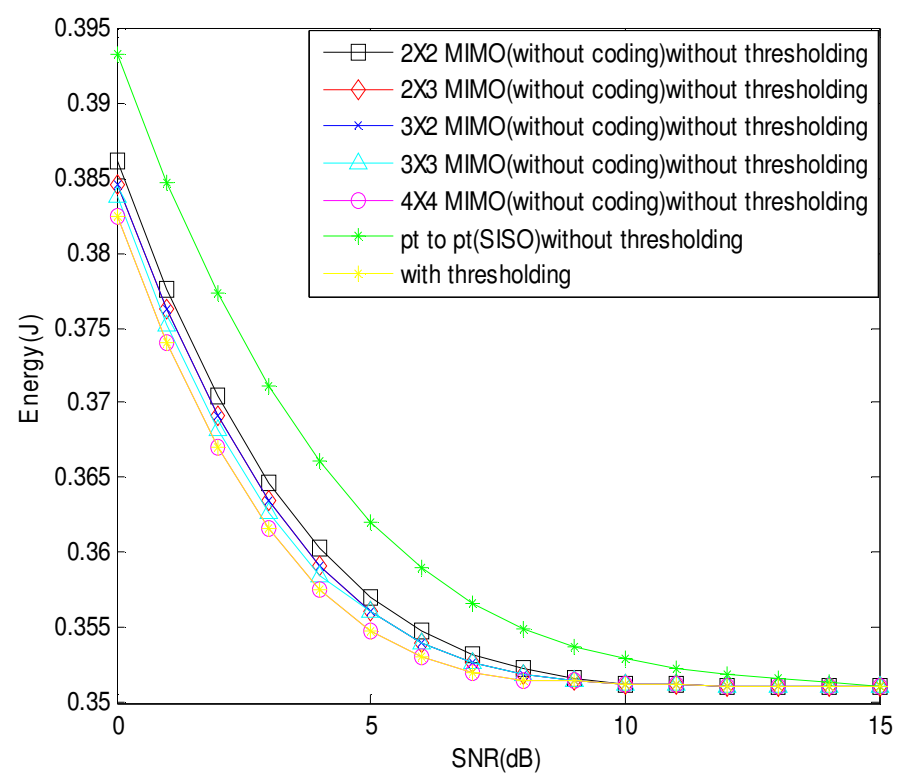

Fig.5. Energy consumption of uncoded scheme for fixed size MIMO configurations and cooperative threshold

\subsection{Performance Analysis of STBC MIMO Scheme}

Similar graphs as that of uncoded schemes are obtained shown in Fig.7 and Fig.8 for energy consumption and delay with STBC coding technique for various sending and receiving group size with and without thresholding scheme. For STBC coding $4 \times 4$ is the dynamic group size selected with cooperative threshold as it incurs lesser energy and delay values. The reduction in energy consumption and delay for $4 \mathrm{x} 4$ group size MIMO configurations are $15 \%$ and $50 \%$ respectively when compared with point to point communication. This is due to the diversity gain exploited by the use of STBC coding techniques. 


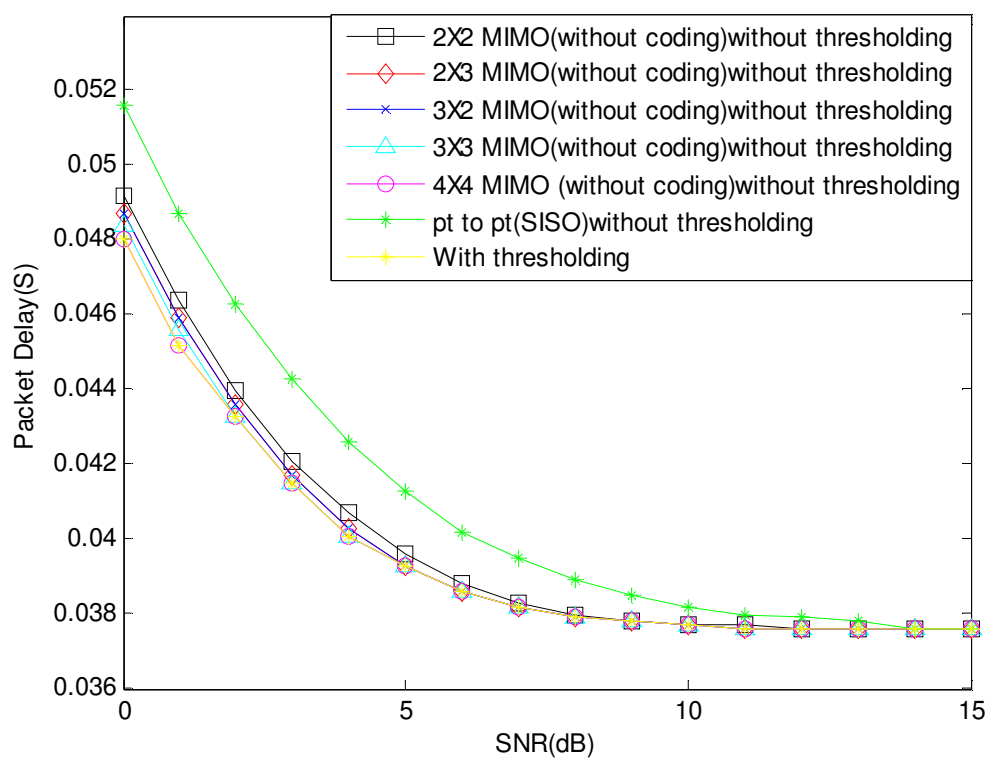

Fig.6. Packet delay of uncoded scheme for fixed size MIMO configurations and cooperative threshold

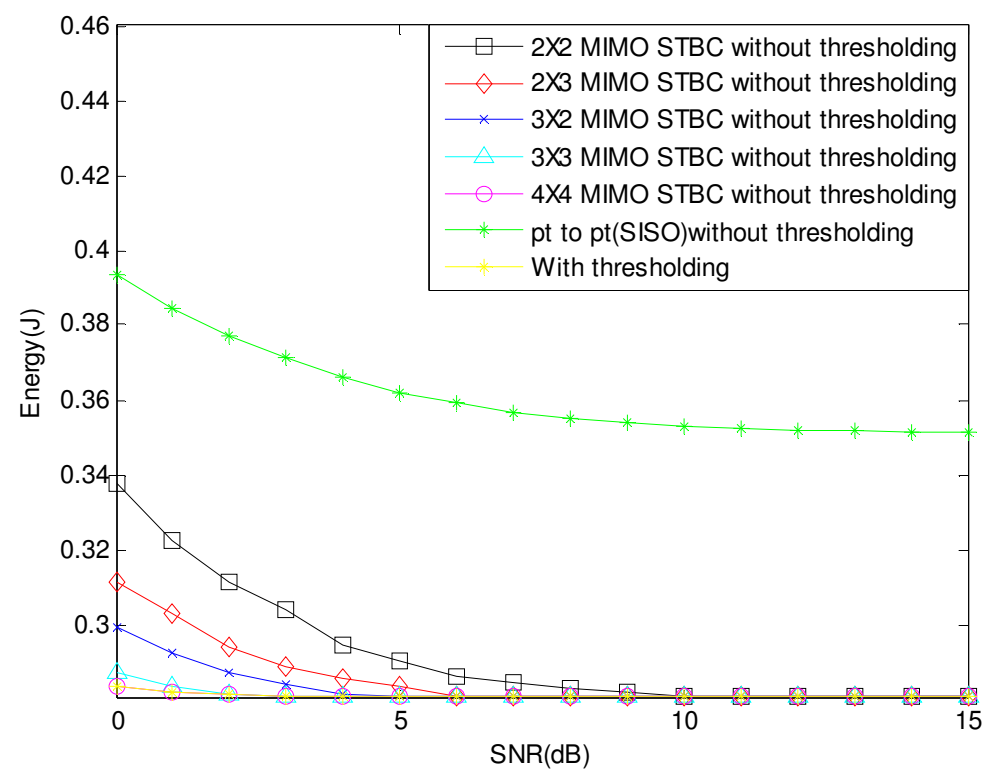

Fig.7. Energy consumption using STBC scheme for various MIMO configurations and cooperative threshold

\subsection{Performance Comparison of Uncoded MIMO and STBC MIMO Scheme}

The performance of uncoded system and STBC MIMO with cooperative threshold is shown in Figs.9 and 10. In case of uncoded system the energy consumption is larger than STBC coding by about $10 \%$. Furthermore, the packet delay of uncoded scheme is $40 \%$ more than the coded scheme. This is due to higher SER value with uncoded scheme. The use of coding technique 
reduces the error in data transmission leading to significant reduction in energy consumption and delay.

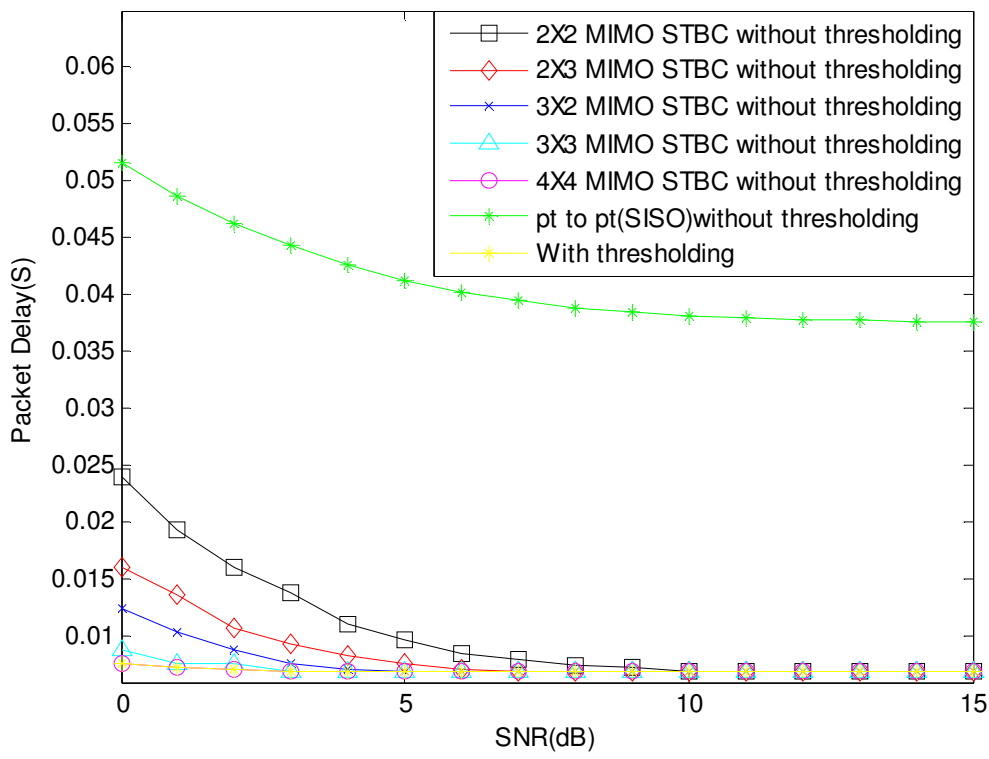

Fig.8. Packet delay using STBC scheme for various MIMO configurations and cooperative threshold

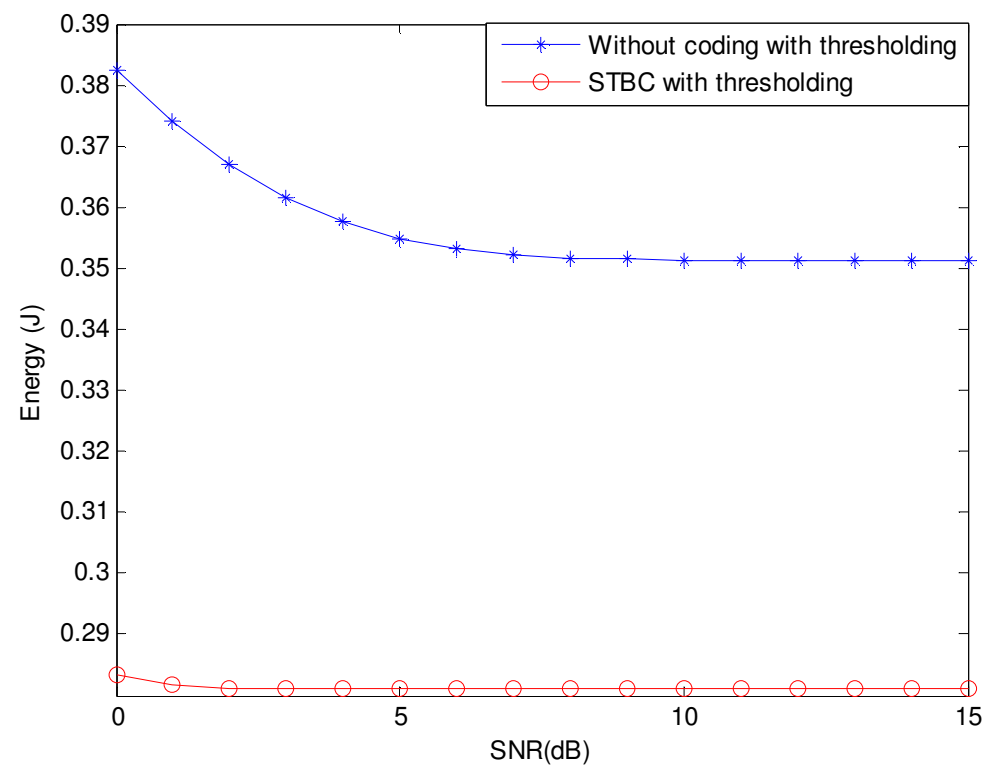

Fig.9. Comparison of energy performance of uncoded scheme and STBC coding with cooperative threshold 


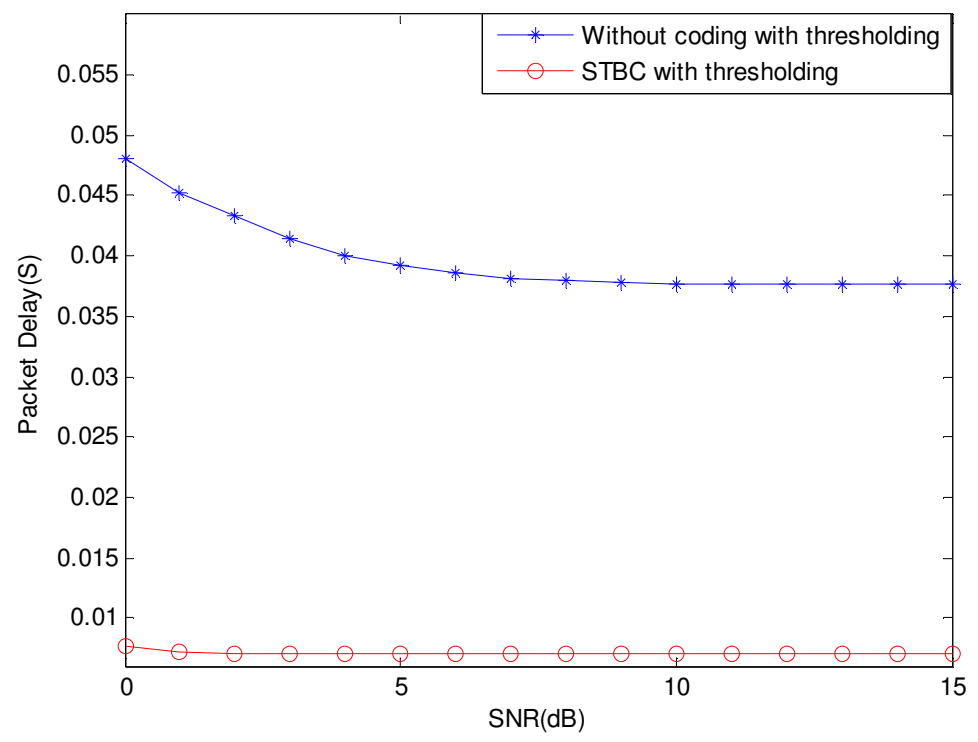

Fig.10. Comparison of delay performance of uncoded scheme and STBC coding with cooperative threshold

\section{Conclusion}

A new cooperative MIMO MAC protocol with dynamic thresholding for WSNs has been presented to maximise the network life time. Transmissions in the proposed protocol proceed only when the expected transmission error rate is less than the cooperative threshold and sending and receiving group sizes are selected to achieve minimum energy consumption and delay. The performance of the proposed MAC protocol is evaluated for fixed uncoded and STBC scheme and are compared with the dynamic group size selected by the proposed scheme. The dynamic group size obtained with the cooperative threshold is $4 \times 4$. Simulation results prove that STBC performs better and consumes 10\% less energy for packet transmissions than uncoded scheme with cooperative threshold. The delay incurred in data transmission with uncoded scheme is $40 \%$ more than MIMO MAC protocol utilising STBC. This significant reduction in delay and energy results from the diversity gain and lesser error probability rates achieved with the coded MIMO systems.

\section{ACKNOWLEDGEMENTS}

The authors would like to express their gratitude to Ms.K.Alamelu, Ms.S.Divyanka and Ms.S.Shobana, under graduate students of Pondicherry Engineering College, Pondicherry for their kind help.

\section{REFERENCES}

[1] Akyildiz. L., Su. W., Sankarasubramanian. Y and Cayirci. E., (2002) "A survey on sensor networks”, IEEE Communications Magazine, Vol. 40, No. 8, pp102-114.

[2] Bravos. G. N. and Efthymoglou. G., (2007) "MIMO-based and SISO multihop sensor network: Energy efficiency evaluation", Proceedings of IEEE International Conference on Wireless and Mobile Computing, Networking and Communications, pp13-18. 
[3] Cui. S., Goldsmith. A. J. and Bahai. A., (2004) "Energy-efficiency of MIMO and cooperative MIMO techniques in sensor networks", IEEE Journal on Selected Areas in Communications, Vol. 22, No. 6, pp1089-1098.

[4] Jayaweera. S. K., (2004) "Energy analysis of MIMO techniques in wireless sensor networks", Proceedings of Annual Conference on Information Sciences and Systems, Princeton, NJ.

[5] Yuan.Y, He. Z. and Chen. M., (2006) "Virtual MIMO- based cross-layer design for wireless sensor networks", IEEE Transactions on Vehicular Technology, Vol.55, No.3, pp856-864.

[6] Yang. H, Shen. H.Y. and Sikdar. B., (2007) "A MAC protocol for cooperative MIMO transmissions in sensor networks", Proceedings of IEEE Global Communications Conference, Exhibition and Industry Forum, Washington, USA, pp636-640.

[7] Yuan. Y., Chen. M. and Kwon. T., (2006) “A novel cluster-based cooperative MIMO scheme for multi-hop wireless sensor networks", EURASIP Journal on Wireless Communication and Networking, Article ID 72493, pp1-9.

[8] Wenqing Cheng, Kanru Xu, Wei Liu, Zongkai Yang and Zheng Feng, (2006) "An energyefficient cooperative MIMO transmission scheme for wireless sensor networks", Proceedings of International Conference on Wireless Communication, Networking and Mobile Computing, pp14.

[9] Shuang Li, Raghu Kishore, Cong Liu and Alvin Lim, (2010) "Efficient multi-path protocol for wireless sensor networks", International Journal of Wireless and Mobile Networks, Vol.2, No.1, pp109-131.

[10] Shinya Ito and Kenji Yoshigoe, (2009) "Performance evaluation of consumed energy-typeaware routing (CETAR) for wireless sensor networks", International Journal of Wireless and Mobile Networks, Vol.1, No.2, pp90-101.

[11] Irfan Ahmed, Mugen Peng, Wenbo Wang and Syed. I. Shah, (2009) "Joint rate and cooperative MIMO scheme optimization in wireless sensor networks", Elsevier Journal on Computer Communications, Vol.32, pp1072-1078.

[12] Hsin-Yi Shen, Haiming Yang, Biplab Sikdar and Shivkumar Kalyanaraman, (2008) “A distributed system for cooperative MIMO transmissions", Proceedings of IEEE Global Communications Conference, Los Angles, USA, pp1-5.

[13] Hsin-Yi-Shen, Haiming Yang and Shivkumar Kalyanaraman, (2008) "Energy efficient cooperative MIMO systems”, Proceedings of IEEE Infocom, Phoenix, USA.

[14] Mehta.S and Kwak. K.S, (2010) "A Power efficient back-off scheme for wireless sensor networks”, International Journal of Wireless and Mobile Networks, Vol.2, No.1, pp15-22.

[15] Li. X., Chen. M., and Liu. W., (2005) "Application of STBC-encoded cooperative transmissions in wireless sensor networks", IEEE Signal Processing Letters, Vol.12, No.2, pp134-137.

[16] Tarokh. V., Jafarkhani. H. and Calderbank. R., (1999) "Space-time block codes from orthogonal designs”, IEEE Transactions on Information Theory, Vol. 45, No. 5, pp1456-1467.

[17] Mohinder Janakiraman, (2004) Space time codes and MIMO systems, Boston, London, Artech House Publishers.

[18] Ahmad. M. R., Dutkiewicz. E. and Huang.X., (2008) "Performance analysis of MAC protocol for cooperative MIMO transmissions in WSN", Proceedings of International Symposium on Personal, Indoor and Mobile Radio Communications, France, pp580-585. 


\section{Authors}

J. Vidhya received B.E degree in Electronics and Communication Engineering from Bharathidasan University, Trichy in 1999 and M.Tech degree in Electronics and Communication Engineering from Pondicherry Engineering College, Pondicherry in 2001. She is pursuing her Ph.D. programme in Department of Electronics and Communication Engineering, Pondicherry University. She is currently working as Assistant Professor in the Department of Electronics and Communication Engineering at Rajiv Gandhi College of Engineering and Technology affiliated to Pondicherry University. Her research interests include computer networks, wireless ad hoc and sensor networks.

P. Dananjayan received Bachelor of Science from University of Madras in 1979, Bachelor of Technology in 1982 and Master of Engineering in 1984 from the Madras Institute of Technology, Chennai and Ph.D. degree from Anna University, Chennai in 1998. He is working as Professor in the Department of Electronics and Communication Engineering, Pondicherry Engineering College, Pondicherry, India. He has been also as a visiting professor to AIT, Bangkok. He has more than 60 publications in National and International Journals. He has presented more than 130 papers in National and International Conferences. He has guided 9 Ph.D candidates and is currently guiding 6 Ph.D students. His research interests include spread spectrum techniques, wireless communication,

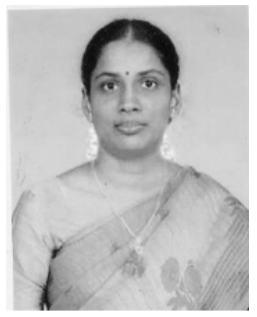
wireless adhoc and sensor networks.

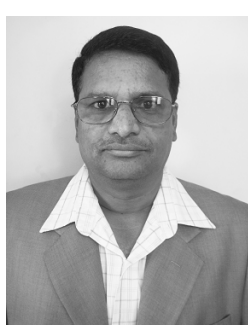

